\title{
DEVELOPMENT OF TURBULENT WAKES EVOLVING FROM ASYMMETRIC SHEAR LAYERS
}

\author{
M. Dghim $\dagger, \ddagger,{ }^{*}$, L. Momayez $\dagger$, M. Ferchichi $\dagger$ and H. Peerhossaini $\S$ \\ $\nmid$ Royal Military College of Canada, Kingston, Ontario, Canada \\ łUniversité de Sherbrooke, Sherbrooke, Québec, Canada \\ $\S$ Université Paris Diderot, Sorbonne Paris Cité, Paris, France \\ E-mail*: Marouen.Dghim@rmc.ca
}

\begin{abstract}
This paper reports an experimental investigation on the response of a turbulent wake past an elongated flat plate to various upstream flow conditions. Different tripping wires were placed on the upper side of the plate downstream the leading edge resulting in asymmetric shear layers evolving from the trailing edges. Mean flow and turbulent fields of the asymmetric wakes were compared to their symmetrical counterpart. The symmetrical wake was found to attain a self-similar state whereas asymmetric wakes continued to slowly evolve towards an expected asymptotic behaviour at a rate that strongly depends on the imposed initial conditions.
\end{abstract}

\section{INTRODUCTION}

The turbulent flow in the wake of wakegenerating bodies has been intensely investigated in the literature due to its occurrence in a wide range of applications, such as aeronautical engineering, heat exchangers, turbomachinery, etc. Such a flow is generally dominated by large scale, coherent structures known as the Von-Karman vortex street in the wake. Much of our current understanding of wake flows is reffered to the studied reported in [1,2]. The effects of flow initial conditions on wake turbulence statistics have also been investigated in a number of papers in order to evaluate the extent to which experimental measurements agreed with self-similar solutions. These studies suggested that the mean flow and the turbulent statistics become independent of the streamwise position when normalized with typical wake velocity and length scales $[3$. One the most comprehensive studies on wake flows, reported in [4, suggested that the normalized turbulent fluctuations, characteristic velocity and length scales

\section{NOMENCLATURE}

$\begin{array}{ll}c & {[\mathrm{~m}]} \\ d_{w} & {[\mathrm{~mm}]} \\ h & {[\mathrm{~m}]} \\ H & {[-]} \\ f & {[\mathrm{~Hz}]} \\ f_{v} & {[\mathrm{~Hz}]} \\ f_{s p} K & {[\mathrm{~Hz}]} \\ S t_{v} & {[-]} \\ R e_{c} & {[-]} \\ R e_{\theta} & {[-]} \\ w(x) & {[\mathrm{m}]} \\ y_{50 \%} & {[\mathrm{~m}]} \\ U & {[\mathrm{~m} / \mathrm{s}]} \\ U_{d} & {[\mathrm{~m} / \mathrm{s}]} \\ U_{\infty} & {[\mathrm{m} / \mathrm{s}]} \\ u^{\prime} & {[\mathrm{m} / \mathrm{s}]} \\ v^{\prime} & {[\mathrm{m} / \mathrm{s}]} \\ E_{u^{\prime} u^{\prime}} & {[\mathrm{m} / \mathrm{s}]} \\ (x, y) & {[\mathrm{m}]}\end{array}$

\author{
chord length of the flat plate \\ diameter of the tripping wire \\ thickness of the flat plate trailing edge \\ boundary layer shape factor \\ frequency \\ von Kármán vortex shedding frequency \\ secondary peak frequency \\ von Kármán Strouhal number \\ Reynolds number based on the chord length \\ Reynolds number based on the momentum \\ thickness \\ wake width \\ transverse location of the half-wake width \\ local mean streamwise velocity \\ local maximum velocity defect \\ freestream velocity \\ local streamwise velocity fluctuations \\ local transverse velocity fluctuations \\ local transverse velocity fluctuations \\ Cartesian coordinate system attached to \\ the flat plate trailing edge
}

Special characters

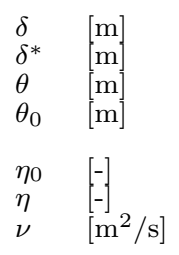

boundary layer thickness

boundary layer displacement thickness

boundary layer momentum thickness

boundary layer momentum thickness

of the ST case

similarity variable of the ST case

similarity variable of the ATT cases

kinematic viscosity

Abbreviations

$\begin{array}{ll}B L & \text { boundary layer } \\ S T & \text { symmetric turbulent-turbulent case } \\ A T T & \text { asymmetric turbulent-turbulent cases }\end{array}$

downstream of the wake generating body depend on the initial conditions. In the same study, it was found that, although the wake mean velocity profiles were self-similar for different wake-generating bodies, different spreading rates were reported in each case. These results have been corroborated by [5] 8 . The influence of the state of the boundary layer at the trailing edge of an elongated bluff body has been well documented experimentally 9, 10. In these studies, it has been suggested that that increas- 
ing the thickness of the boundary layer alters the base pressure of the wake, moves the stagnation point closer to the base, affects the shape of the velocity profile, induces lower centreline velocities and results in a wider wake. Roos 11] and Thomas and Liu 12, although they considered an initially asymmetric wake, they focused mainly on the effect of pressure gradient on the wake development. Recently, Kim et al. 13] conducted an experimental study on the effect of the boundary layer asymmetry on the near wake of a profiled flat plate and they reported that the asymmetry of the initial conditions affected the location of the maximum mean velocity deficit. In their study, only the near wake development of a laminar-laminar, laminar-transitional and laminar-turbulent upstream conditions were examined. Momayez et al. 14] considered laminar-laminar, laminarturbulent and turbulent-turbulent asymmetric boundary layers with focus on the wake dynamics and the convection velocity of the VonKarman vortex street. They reported that the wake of the laminar-turbulent case was the most largely effect by the asymmetry of the initial conditions. It has been documented that the higher turbulent statistics response to initial conditions is very slow, and thus generally depart from the self-similar behaviour. For asymmetric wakes, there is still a need for detailed data to further elucidate the physical reasons that make the wake strongly dependent on initial flow asymmetry. The present work will attempt to provide additional insights on the subject by performing $2 \mathrm{D}$ measurements in the turbulent wake of a flat plate evolving from asymmetric boundary layers at the trailing edge of an elongated flat plate. As opposed to previous experimental studies, in this work, the free-stream conditions and the pressure gradient were maintained constant while the degree of asymmetry of the shear layers was gradually increased. A total of six cases were experimentally studied; turbulent-turbulent symmetric wake (ST) and turbulent-turbulent asymmetric wakes (ATT) with five different initial conditions imposed at the trailing edge of the flat plate. It is worth noting that the asymmetric turbulent-turbulent wake case has not been addressed in the literature.

\section{EXPERIMENTAL APPARATUS AND MEASUREMENT TECH- NIQUES}

The experiments were carried out in the low speed wind tunnel of the Department of
Mechanical and Aerospace Engineering at the Royal Military College of Canada. It has a square cross section of $0.41 \times 0.41 \mathrm{~m}^{2}$ and test section of $4 \mathrm{~m}$ length. The wake generating model, shown in Figure 1, consisted of a thick trailing edge flat plate mounted parallel to the flow at the center of the test section. The flat plate was made of aluminium and was mirror-finished. It had a chord length, $c$, of $0.508 \mathrm{~m}$, a width, $w$, of $0.381 \mathrm{~m}$ and a thickness, $h$, of $0.0127 \mathrm{~m}$ resulting in a blockage ratio of about $3 \%$. The width of the flat plate spanned the wind tunnel width to ensure two dimensionality of the flow. To minimize the effect of the separation-attachment inherent blunt-shaped leading edge, an ellipticshaped leading edge, manufactured from ABS plastic, was carefully attached to the flat plate such that the surface transition from the leading edge to the flat plate was reasonably smooth. The leading edge was covered with a thin aluminium film. The Cartesian coordinate system $(x, y, z)$ adopted for this study was anchored at the center of the flat plate trailing edge at $h / 2 . x, y$ and $z$ denote the streamwise, transverse, and spanwise spatial coordinates, respectively. The upstream conditions considered for this study were experimentally investigated at a Reynolds number, $R e_{c}$, based on the freestream velocity, $U_{\infty}$, and the flat plate chord length, $c$, of about $5.5 \times 10^{5}$. A total of six asymmetric initial conditions were achieved by perturbing the boundary layer developing on the upper side of the flat plate using aluminium cylindrical wires with diameters, $d_{w}$, of $0.36,0.54$, $0.80,1.20$ and $1.54 \mathrm{~mm}$, respectively. The tripping wires were placed at the location of the junction between the leading edge and the flat plate to eliminate any effects related to surface transition. A miniature, custom-built L-shaped hotwire probe (Auspex Scientific) along with a multi-channel constant temperature anemometer (TSI IFA300) were used to measure the boundary layer profiles close to the trailing edge at $x / c=0.98$. The smallest separation distance between the hotwire and the flat plate surface was about $0.4 \mathrm{~mm}$, thus ensuring a reasonable spatial resolution in the measurement of the boundary layer parameters. In the wake region, mean flow and turbulent statistics were measured using a miniature cross-wire probe (Auspex scientific, AHWX-100) with tungsten sensors having a length of $1 \mathrm{~mm}$ diameter of $5 \mu \mathrm{m}$. Both hotwire probes were mounted on a streamlined support extending from an external traversing system. The latter allowed for the probes to travel in both streamwise and 
transverse directions with a spatial of resolution of $0.127 \mu \mathrm{m}$. The acquired signals were low-pass filtered at a frequency of $2 \mathrm{KHz}$ and were sampled at $5 \mathrm{KHz}$ using a 16 -bit A/D converter (IOTECH Wavebook 516). To ensure fully converged turbulent statistics, sufficiently long records of $52.4 \mathrm{~s}$ and $209.7 \mathrm{~s}$ were acquired for turbulence and spectra measurements, respectively.

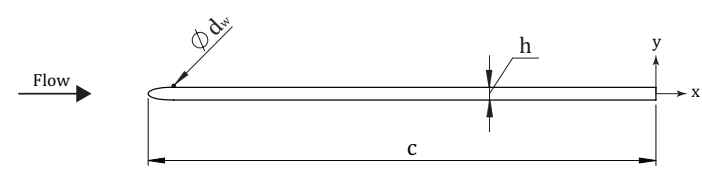

Figure 1: Experimental setup.

Table 1: Boundary layer parameters at $x / c=$ 0.98 .

\begin{tabular}{cccccc}
\hline & $\delta$ & $\delta^{*}$ & $\theta$ & $H$ & $R e_{\theta}$ \\
\hline \hline$S T$ & 6.49 & 0.70 & 0.42 & 1.66 & 473 \\
$d_{w}{ }^{\dagger}=0.36$ & 8.30 & 1.00 & 0.67 & 1.49 & 743 \\
$d_{w}{ }^{\dagger}=1.20$ & 10.60 & 1.51 & 1.10 & 1.37 & 1092 \\
$d_{w}{ }^{\dagger}=1.54$ & 11.80 & 1.70 & 1.30 & 1.30 & 1238 \\
\hline
\end{tabular}

$\dagger$ wire diameters are in $\mathrm{mm}$.

\section{RESULTS AND DISCUSSION}

\section{Upstream Flow Development}

In the present work, initial conditions were imposed by modifying the boundary layer (BL) characteristics as it reached the trailing edge of the upper surface of the flat plate. The BL thickness, $\delta$, displacement thickness, $\delta^{*}$, momentum thickness, $\theta$, the shape factor, $H$, and the Reynolds number based on the momentum thickness, $R e_{\theta}=U_{\infty} \theta / \nu$, were evaluated at $x / c=0.98$ and were summarized in Table 1 for the different cases investigated. At the trailing edge of the flat plate, the measured shape factors, $H$, of both natural and tripped BLs ranged from 1.66 to 1.30 , typical of turbulent BLs. The thicknesses of the shear layers developing on both sides of the trailing edge, were dictated by the momentum thickness, $\theta$, of the BLs at $x / c=0.98$. For the natural BL, the momentum thicknesses, $\theta_{0}$, were similar on both sides of the flat plate. However, for tripped cases, a ratio, $\theta / \theta_{0}$, between $\theta$ of the thicker (upper) $\mathrm{BL}$ and $\theta_{0}$ of the thinner (lower) BL was used to measure the degree of asymmetry at which the wake shear layers evolved from the trailing edges.

\section{Natural Wake}

Profiles of the local velocity defect, $U_{e}-\bar{U}$, scaled by the local velocity defect at the wake centreline, $U_{d}$, were plotted against the selfsimilarity variables $\eta_{0}=y / w(x)$ for the ST wake in Figure 2. The wake width, $w(x)$, was defined as the distance separating the transverse positions, $\pm y_{50 \%}$, with respect to the wake centerline where the local scaled velocity defect drops to 0.5 [14. Profiles of the scaled velocity deficit of the ST case showed the universal wake-like behaviour with an excellent collapse at all downstream positions indicating a well-established self-preserved state. The profiles also showed reasonable agreement with the asymptotic expression proposed in [4]:

$$
\frac{U_{e}-\bar{U}}{U_{d}}=\exp \left(-0.637 \eta_{0}^{2}-0.056 \eta_{0}^{4}\right)
$$

In Figure 3, the normalized wake widths, $w(x) / h$, of the ST wake were found to grow with $x^{\sim 0.5}$ in agreement with the similarity analysis of [15] and the experiments reported in 44, 8, given a sufficient distance downstream the trailing edge of the flat plate [16].

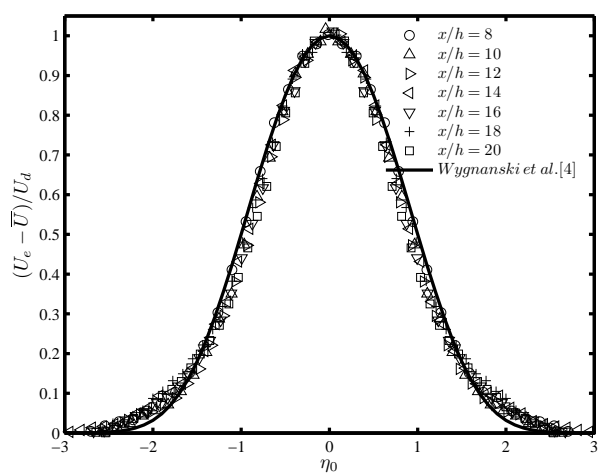

Figure 2: Streamwise development of the normalized wake width for the different cases studied.

Transverse profiles of the streamwise Reynolds stress, $\sqrt{{u^{\prime}}^{2}}$ scaled in the traditional manner by the local maximum velocity defect, $U_{d}$, are illustrated in Figure 4a for the ST case. It can be inferred from this figure that the traditional scaling resulted in a reasonable collapse of the profiles of $\sqrt{{\overline{u^{\prime}}}^{2}}$ at all downstream positions. The collapsed profiles were symmetrical with respect to the wake centreline where peak values of $\sqrt{u^{\prime 2}}$ are located. Although not shown here, the two distinct peaks in the transverse profiles 


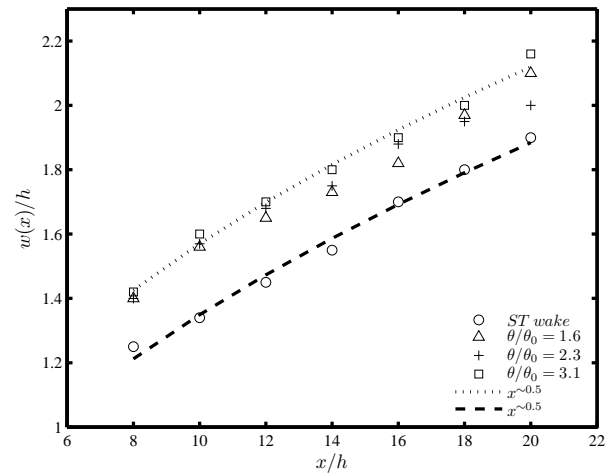

Figure 3: Streamwise development of the normalized wake width for the different cases studied.

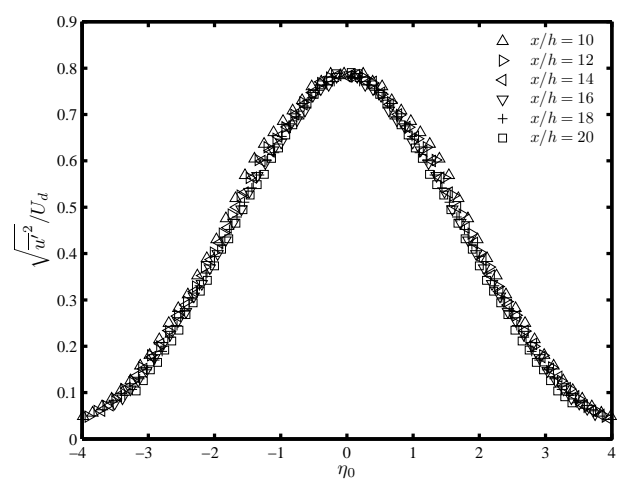

(a)

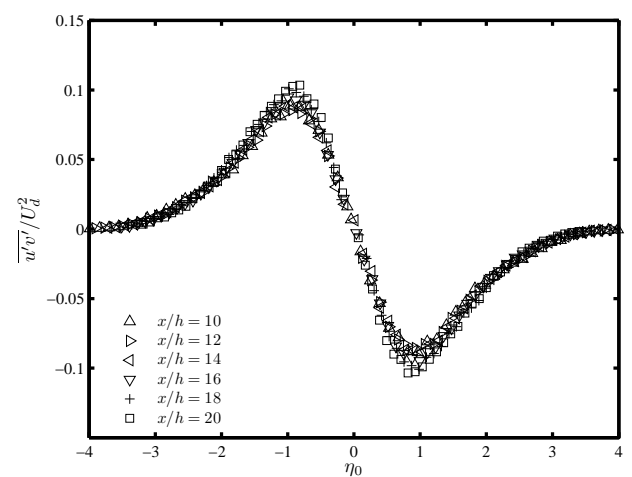

(b)

Figure 4: Self-similar profiles of (a) $\left(U_{e}-\bar{U}\right) / U_{d}$, (b) $\sqrt{u^{\prime 2}} / U_{d}$ and (c) $\overline{u^{\prime} v^{\prime}} / U_{d}^{2}$ of the $\mathrm{ST}$ wake.

of $\sqrt{{\overline{u^{\prime}}}^{2}}$ where maximum shear occurs (usually seen in the near wake), disappeared at large downstream distance as the wake attained an asymptotic state in agreement with [17, 18. A similar collapse was also observed in the profiles of the Reynolds shear stress, $\overline{u^{\prime} v^{\prime}}$, scaled by $U_{d}^{2}$ as shown in Figure 4b. The profiles also exhib- ited a symmetrical distribution with respect to the wake centreline at all downstream positions with peak values located in the region of maximum velocity gradients in the transverse direction. The self-similar behaviour of the secondorder turbulent statistics indicates that the turbulent field evolved with the same manner as the mean flow (Figure 2) suggesting that selfsimilarity of the ST case was complete.

\section{Asymmetric Wakes}

It has been reported that wakes tend to move toward the perturbed boundary layer [11 13. This behaviour was clearly shown in the profiles of the scaled velocity defect plotted against the similarity variable $\eta=\left(y-y_{0}\right) / w(x)$ as illustrated in Figures [5a to [5c] Here $y_{0}$ denotes the transverse position of the maximum velocity defect. The profiles of $\left(U_{e}-\bar{U}\right) / U_{d}$ showed a noticeable departure from the asymptotic fit given in equation 1 in the outer region of the wake with the effect being more pronounced for $\eta>1$. In the central wake region, however, profiles of the scaled velocity defect exhibited a better collapse. Furthermore, at a given streamwise position, the increased asymmetry of the initial conditions was shown to increase the discrepancy between the self-similar profiles of $\left(U_{e}-\bar{U}\right) / U_{d}$ and the asymptotic fit given in equation 1 as evidenced in Figure 6. A systematic asymmetry in the ATT profiles towards the perturbed side were shown to increase with increased $\theta / \theta_{0}$. In Figure 3, the normalized wake widths, $w(x) / h$, of the ATT cases exhibit virtually an identical streamwise growth to that of the ST wake. However, at all downstream locations, the wake widhts of the ATT wakes were larger than those of the ST wake indicating an increased spreading of the ATT wakes with increased asymmetry. For the ATT cases, profiles of $\sqrt{{\overline{u^{\prime}}}^{2}} / U_{d}$, shown in Figures $7 \mathrm{a}$ to $7 \mathrm{c}$. exhibited a noticeable asymmetry with respect to the wake centreline $(\eta=0)$ as opposed to the ST case. In that, the transverse locations of the local peaks of $\sqrt{u^{\prime 2}} / U_{d}$ appeared to shift towards to perturbed side with decreased peak values as $\theta / \theta_{0}$ increased indicative of higher turbulent mixing in the perturbed side of the ATT wakes. Similarly, profiles of $\overline{u^{\prime} v^{\prime}} / U_{d}^{2}$ showed a distinctly asymmetric behaviour with increased $\theta / \theta_{0}$ as illustrated in Figures 8a to 8c Additionally, peaks of $\overline{u^{\prime} v^{\prime}} / U_{d}^{2}$ appeared on the perturbed side with values reaching nearly one order of magnitude higher than that of the the non-perturbed side for $\theta / \theta_{0}=3.10$; this reflects the strong influence of the wake asymmetry on 


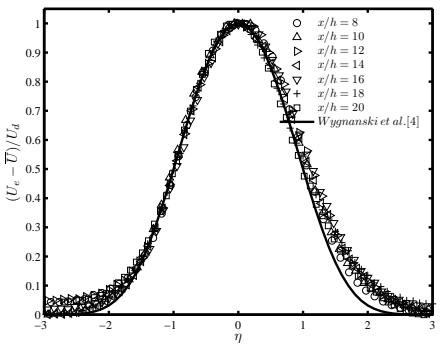

(a) $\theta / \theta_{0}=1.6$

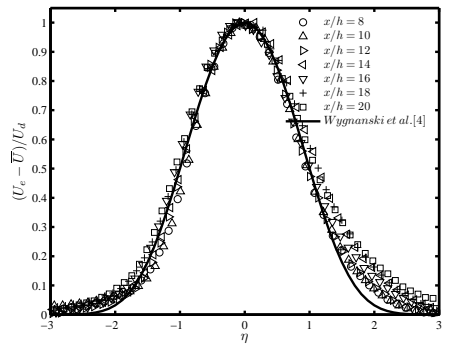

(b) $\theta / \theta_{0}=2.3$

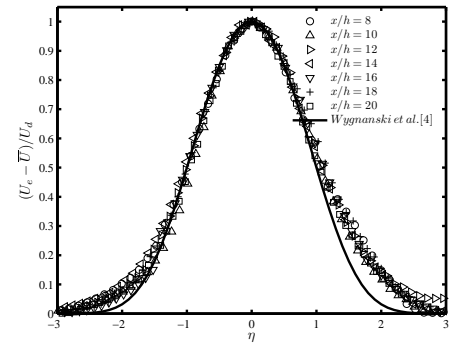

(c) $\theta / \theta_{0}=3.1$

Figure 5: Profiles of the scaled velocity defect for different initial conditions, $\theta / \theta_{0}$.

the evolution of the turbulent field. Furthermore, for the ATT cases, the traditional scaling adopted in the ST case failed to collapse the profiles of $\sqrt{u^{\prime 2}}$ and $\overline{u^{\prime} v^{\prime}}$ particularly at high $\theta / \theta_{0}$. As seen in the scaled mean flow profiles (Figures 2 and 5a to 5c), the lack of collapse in the perturbed side was clearly highlighted in the profiles of $\overline{u^{\prime} v^{\prime}} / U_{d}^{2}$, indicating that the similarity of the asymmetric cases was incomplete and that their wakes were still evolving slowly in the streamwise direction towards a well-established asymptotic state [19].

\section{Spectral Analysis}

Power spectra of the streamwise velocity fluctuations, $E_{u^{\prime} u^{\prime}}$, measured at carefully selected positions in the upper side of both ST and ATT wakes, were illustrated in Figure 9. For readability, only spectra of the ATT wakes at $\theta / \theta_{0}$ of 2.3 and 3.1 were shown. For the ST wake, the vortex shedding frequency, $f_{v}$, was evidenced by the large peak observed at a frequency of about $355 \mathrm{~Hz}$ corresponding to a von Kármán Strouhal number, $S t_{v}=f_{v} h / \bar{U}_{\infty}$, of 0.24 in close agreement with the values reported in 20. Higher frequency peaks observed in the energy spectrum of the ST wake correspond to the subharmonics of the vortex shedding frequency, $f_{v}$, typical of a turbulent wake flow. When the upper BL was perturbed, the vortex shedding frequencies of the ATT wakes exhibited a slight shift towards lower frequencies corresponding to values of $S t_{v}$ of about 0.21 and 0.20 for $\theta / \theta_{0}=2.3$ and $\theta / \theta_{0}=3.1$, respectively. The reduction in the von Kármán $S t_{v}$ was attributed to the increased turbulence level in the wake of the ATT cases in agreement with 21. A similar shift was also observed in the subharmonics of the vortex shedding frequency $f_{v}$ of the ATT wakes. Interestingly, large secondary peaks appeared in the spectra of the ATT wakes at frequency values, $f_{s p}$, measured to be $12 \%$

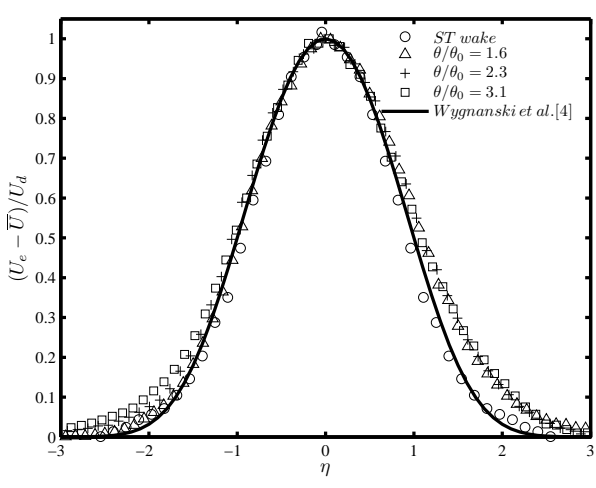

Figure 6: Profiles of the scaled velocity defect for different $\theta / \theta_{0}$ at $x / h=14$.

lower than their respective $f_{v}$. These secondary peaks might be attributed to the low-frequency instability induced by the the shrinkage and enlargement of the recirculation zone as suggested in [22]. Additionally, the energy level associated with the low frequency fluctuations was found to increase with increased wake asymmetry. At higher frequencies, peaks of the vortex shedding subharmonics of the ATT wakes became broader and indiscernible due to the increased turbulence in the perturbed side of the ATT wakes. Conversely, the energy level associated with the high frequency fluctuations was found to increase with increased turbulence in the ATT wakes in agreement with [23].

\section{CONCLUSION}

This work reports an experimental investigation on the effect of asymmetric shear layers on the turbulent wake of an elongated flat plate. The asymmetry of the shear layers at the trailing edge of the flat plate was achieved by tripping the upper boundary layer. In the case of the symmetrical wake, profiles of the mean flow and the turbulent field collapsed in similarity 


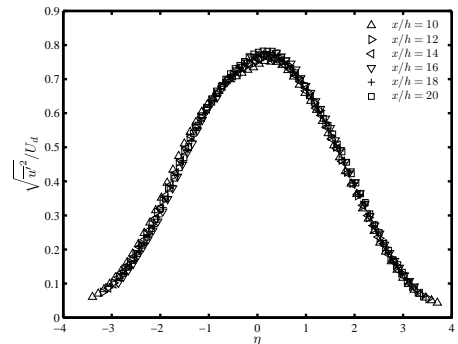

(a) $\theta / \theta_{0}=1.6$

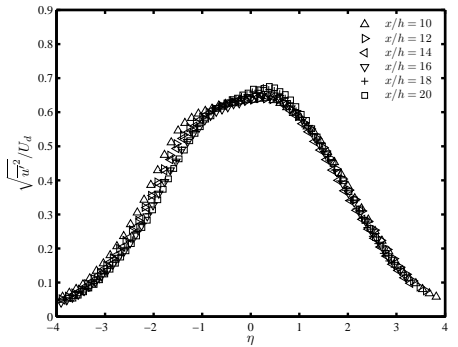

(b) $\theta / \theta_{0}=2.3$

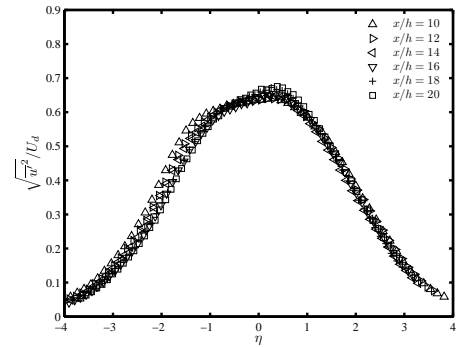

(c) $\theta / \theta_{0}=3.1$

Figure 7: Profiles of the scaled streamwise Reynolds stress for different initial conditions, $\theta / \theta_{0}$.

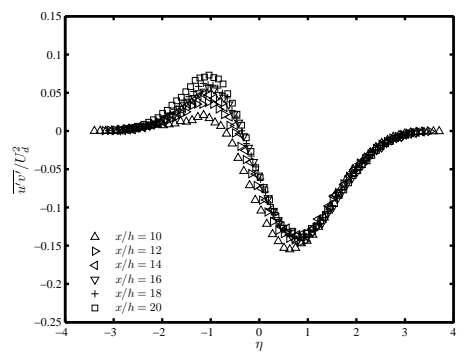

(a) $\theta / \theta_{0}=1.6$

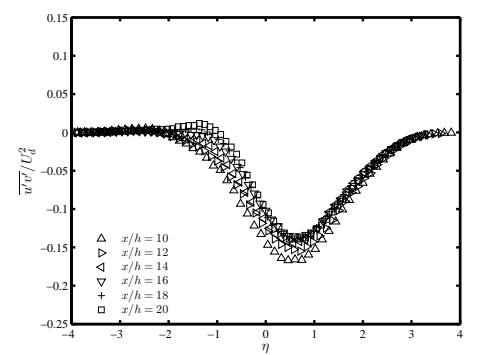

(b) $\theta / \theta_{0}=2.3$

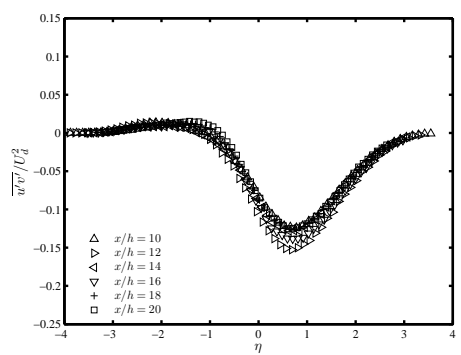

(c) $\theta / \theta_{0}=3.1$

Figure 8: Profiles of the scaled Reynolds shear stress for different initial conditions, $\theta / \theta_{0}$.

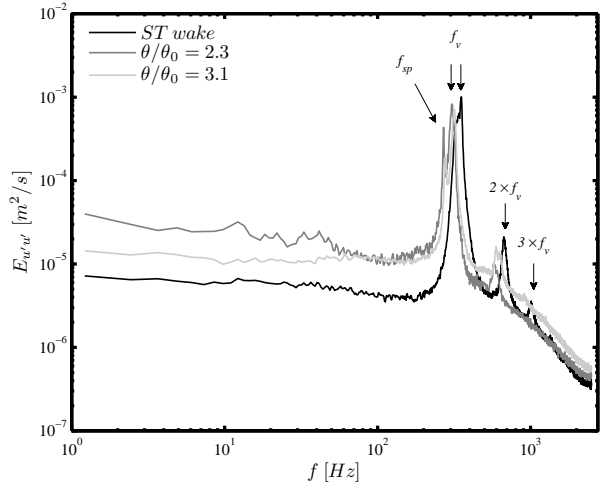

Figure 9: Spectra of $u^{\prime}$ at $x / h=1$ and $y / h=$ 0.75 .

scaling at all downstream positions indicating a well-established asymptotic state. Asymmetric wakes showed a tendency to a self-similar state at a rate that strongly depends on the imposed initial conditions. Spectra measured in the upper side of the wake revealed the appearance of a second peak in the asymmetric cases with a frequency slightly smaller than that of the vortex shedding frequency. A noticeable increase in the energy level associated with a broadening of the peaks of the vortex shedding subharmonics was clearly observed in the spectra of the asymmetric wakes as a result of the increased turbulence.

\section{Acknowledgement}

The authors gratefully acknowledge the CD ARP fund provided by the Royal Military College of Canada for the support of this research.

\section{References}

[1] H. Grant, "The large eddies of turbulent motion," Journal of Fluid Mechanics, vol. 4, no. 02, pp. 149-190, 1958.

[2] A. Townsend, "The mechanism of entrainment in free turbulent flows," Journal of fluid mechanics, vol. 26, no. 04, pp. 689$715,1966$.

[3] K. Sreenivasan, "Evolution of the centerline probability density function of temperature in a plane turbulent wake," Physics of Fluids (1958-1988), vol. 24, no. 7, pp. 12321234, 1981.

[4] I. Wygnanski, F. Champagne, and B. Marasli, "On the large-scale structures in two-dimensional, small-deficit, turbulent wakes," Journal of Fluid Mechanics, vol. 168, pp. 31-71, 1986. 
[5] Y. Zhou and R. Antonia, "Critical points in a turbulent near wake," Journal of Fluid Mechanics, vol. 275, pp. 59-81, 1994.

[6] S. Ghosal and M. M. Rogers, "A numerical study of self-similarity in a turbulent plane wake using large-eddy simulation," Physics of Fluids (1994-present), vol. 9, no. 6, pp. 1729-1739, 1997.

[7] R. D. Moser, M. M. Rogers, and D. W. Ewing, "Self-similarity of time-evolving plane wakes," Journal of Fluid Mechanics, vol. 367, pp. 255-289, 1998.

[8] X. Liu, F. O. Thomas, and R. C. Nelson, "An experimental investigation of the planar turbulent wake in constant pressure gradient," Physics of Fluids (1994-present), vol. 14, no. 8, pp. 2817-2838, 2002.

[9] C. Przirembel, R. Page, and J. Porteiro, "Modification of subsonic wakes using boundary layer and base mass transfer," AIAA Journal, vol. 21, no. 5, pp. 665-670, 1983.

[10] J. Porteiro and V. Perez-Villar, "Wake development in turbulent subsonic axisymmetric flows," Experiments in fluids, vol. 21, no. 3, pp. 145-150, 1996.

[11] F. W. Roos, "Experimental studies of wake retardation in a simulated high lift system flow field," AIAA paper, no. 97-1813, 1997.

[12] F. O. Thomas and X. Liu, "An experimental investigation of symmetric and asymmetric turbulent wake development in pressure gradient," Physics of Fluids (1994present), vol. 16, no. 5, pp. 1725-1745, 2004.

[13] D.-H. Kim, J.-W. Chang, H.-B. Kim, and M.-H. Sohn, "Upstream condition effects on the evolution of symmetric and asymmetric near-wakes of a flat plate," Aerospace Science and Technology, vol. 14, no. 1, pp. 4955,2010 .

[14] L. Momayez, M. Dghim, M. Ferchichi, and S. Graveline, "Near field development of planar wakes under the effect of asymmetric initial conditions," Journal of Fluids Engineering, vol. 137, no. 9, p. 091201, 2015.

[15] A. A. Townsend, The structure of turbulent shear flow. Cambridge university press, 1956.
[16] H. Schlichting and K. Gersten, Boundarylayer theory. Springer Science \& Business Media, 2003.

[17] R. Chevray and L. S. Kovasznay, "Turbulence measurements in the wake of a thin flat plate.," AIAA Journal, vol. 7, no. 8, pp. 1641-1643, 1969.

[18] B. Ramaprian, V. Patel, and M. Sastry, "The symmetric turbulent wake of a flat plate," AIAA Journal, vol. 20, no. 9, pp. $1228-1235,1982$.

[19] J. M. Jimenez, M. Hultmark, and A. Smits, "The intermediate wake of a body of revolution at high reynolds numbers," Journal of Fluid Mechanics, vol. 659, pp. 516-539, 2010.

[20] Z. Taylor, E. Palombi, R. Gurka, and G. Kopp, "Features of the turbulent flow around symmetric elongated bluff bodies," Journal of Fluids and Structures, vol. 27, no. 2, pp. 250-265, 2011.

[21] Q. Zhang, S. W. Lee, and P. M. Ligrani, "Effects of surface roughness and freestream turbulence on the wake turbulence structure of a symmetric airfoil," Physics of Fluids (1994-present), vol. 16, no. 6, pp. 2044-2053, 2004.

[22] P. Luchini, F. Giannetti, and J. Pralits, "Structural sensitivity of the finiteamplitude vortex shedding behind a circular cylinder," in IUTAM Symposium on Unsteady Separated Flows and their Control, pp. 151-160, Springer, 2009.

[23] I. Khabbouchi, H. Fellouah, M. Ferchichi, and M. S. Guellouz, "Effects of free-stream turbulence and reynolds number on the separated shear layer from a circular cylinder," Journal of Wind Engineering and Industrial Aerodynamics, vol. 135, pp. 46-56, 2014. 